\title{
DIA Solitary and Shock Waves in Dusty Multi-Ion Dense Plasma with Arbitrary Charged Dust
}

\author{
M. S. Zobaer, N. Roy, A. A. Mamun \\ Department of Physics, Jahangirnagar University, Dhaka, Bangladesh \\ Email: niparoybd@gmail.com,niparoybd@yahoo.com
}

Received May 20, 2012; revised June 17, 2012; accepted July 8, 2012

\begin{abstract}
The nonlinear propagation of the DIA (dust ion-acoustic) waves in multi-ion dense plasma system containing degenerate electrons, both positive and negative ions, arbitrary charged dust grains has been investigated by employing the reductive perturbation method. The nonlinear waves (solitary and shock waves) have been observed to be formed in case of both positive and negative charged dust grains from the stationary solution of the Korteweg de-Vries (K-dV) equation and Burger's equation. The fundamental properties of such nonlinear waves have been theoretically analyzed by comparing system potential for both positive and negative dust grains. It has been shown that the basic features of these waves are significantly modified by the positive and negative ions drift speed and polarities of dust grains. The implications of our results in space and laboratory plasmas are briefly discussed.
\end{abstract}

Keywords: Degenerate Pressure; Solitary and Shock Waves; Reductive Perturbation Method; Multi Ion Plasma; Arbitrary Charged Dust

\section{Introduction}

To study the fundamental properties [1-6] of degenerate or extremely dense, which means the denser than ordinary solids, matter has an important role in understanding the electrostatic perturbation existing in extreme conditions [1-6]. Now-a-days the most theoretical concerns are to analysis the environment of the compact objects having their interiors supporting themselves via degenerate pressure. The degenerate pressure, which produces because of the combination effect of Pauli's exclusion principle (Wolfgang Ernst Pauli, 1925) and Heisenberg's uncertainty principle (Werner Heisenberg, 1927), depends only on the number density of constituent particles [7-9], but it does not depend on its own temperature.

Generally in case of considering the compact astrophysical objects, primordial universe, and the process of expanding universe [10-17] which provides us the idea that there may be a possibility to have some heavy elements in addition with lighter elements which are considered by the authors in their previous research paper [7-9]. It has been found the existing plasma waves spectra [18-20] are generally modified because of the presence of the static dust grains. So, in the study of the propagation of nonlinear waves in the plasma system some important features can be observed under consideration of degenerate pressure and thus the dust grains have the vital role in multi-ion dense plasma systems.
Considering this idea we are studying the multi-ion plasma with some additional particles, static dust grains with arbitrary positively and negative charge, to make our present analysis meaningful. This type of plasma is considered as a building block of our early universe [21], and also as an omnipresent ingredient of a number of astrophysical objects such as active galactic nuclei [22], pulsar magnetospheres [23], solar flares [24], fireballs producing $\gamma$-ray bursts [25], etc.

There are already a number of works on waves and instabilities [26-35], quantum effects on linear [30,32,35] and nonlinear [31,33] propagation of electrostatic waves, analyzed by using the quantum QHD (hydrodynamic) model $[26,35]$, and by using the quantum QMHD (magneto-hydrodynamic) model [30-33]. Some others were on nonlinear propagation of electrostatic waves in degenerate quantum plasma [36-38]. Very recently, a number of authors are trying to study the electrostatic perturbation in degenerate plasmas [7-9] where their main concern is to develop the theoretical investigations on the interstellar compact objects, having the density of their interiors becomes extremely high to provide non-thermal pressure through degenerate pressure of their constituent particles and particle-particle interaction.

To the best of our knowledge, still there is no attempt has been made to study the effect of the linear or nonlinear propagation of the unmagnetized electrostatic waves in degenerate multi-ion dusty plasma on the DIA solitary 
and shock waves. Therefore in our present work, we consider a multi-ion dense plasma system with degenerate electrons following the equations of the state valid for both the non-relativistic and ultra-relativistic limits, and ions (both positive and negative) following the non-relativistic limits with two additional charged particles, positively and negatively charged dust grains.

\section{Governing Equations}

We are trying to investigating the propagation of an electrostatic perturbation mode in a degenerate dense multiion dusty plasma containing ultra-relativistic degenerate electrons, non-relativistic degenerate inertial ions having both positive and negative ions and negative dust grains. The dynamics of the electrostatic waves propagating in such a plasma system is governed by

$$
\begin{gathered}
\frac{\partial n_{s}}{\partial t}+\frac{\partial}{\partial x}\left(n_{s} u_{s}\right)=0, \\
\frac{\partial u_{p}}{\partial t}+u_{p} \frac{\partial u_{p}}{\partial x}+\frac{\partial \phi}{\partial x}+\frac{K_{1}}{n_{p}} \frac{\partial n_{p}^{\alpha}}{\partial x}-\eta \frac{\partial^{2} u_{p}}{\partial x^{2}}=0, \\
\frac{\partial u_{n}}{\partial t}+u_{n} \frac{\partial u_{n}}{\partial x}-\beta \frac{\partial \phi}{\partial x}+\frac{K_{1}}{n_{n}} \frac{\partial n_{n}^{\alpha}}{\partial x}-\eta \frac{\partial^{2} u_{n}}{\partial x^{2}}=0, \\
n_{e} e \frac{\partial \phi}{\partial x}-K_{2} \frac{\partial n_{e}^{\gamma}}{\partial x}=0, \\
\frac{\partial^{2} \phi}{\partial x^{2}}=\left(1-p \mu-\alpha_{n}\right) n_{e}-n_{p}+\left(1-p \mu-\alpha_{e}\right) n_{n}+p \mu,
\end{gathered}
$$

where $n_{s}(s=p, n, e)$ is the plasma species number density normalized by its equilibrium value $n_{s 0} \quad\left(n_{e 0}\right)$, $u_{s}$ is the plasma species ion fluid speed normalized by $C_{p m}=\left(m_{e} c^{2} / m_{p}\right)^{1 / 2}$ with $m_{e}\left(m_{p}\right)$ being the electron (plasma ion species) rest mass and $c$ being the speed of light in vacuum, $\phi$ is the electrostatic wave potential normalized by $m_{e} c^{2} / e$ with $e$ being the magnitude of the charge of an electron, the time variable $(t)$ is normalized by $\omega_{p m}=\left(4 \pi n_{0} e^{2} / m_{p}\right)^{1 / 2}$, and the space variable $(x)$ is normalized by $\lambda_{m}=\left(m_{e} c^{2} / 4 \pi n_{0} e^{2}\right)^{1 / 2}$. The coefficient of viscosity $\eta$ is a normalized quantity given by $\omega_{p m} \lambda_{m}^{2} m_{s} n_{s 0}, \beta$ is the ratio of negative and positive ion masses multiplied by their charge per ion, $Z_{j}$ (where $j=p, n$ ), $\mu$ is the ratio the number density of charged dust and ion, and $p$ is positive when we consider the negative dust grains and it is negative when we consider the positive dust grains. The constants $K_{1}=n_{j 0}^{\alpha-1} K_{i} / m_{j} c^{2}$ and $K_{2}=n_{e 0}^{\gamma-1} K_{e} / m_{e} c^{2}$. The equations of state used here are given by

$$
P_{i}=K_{i} n_{i}^{\alpha}
$$

where $\quad \alpha=\frac{5}{3} ; K_{i}=\frac{3}{5}\left(\frac{\pi}{3}\right)^{\frac{1}{3}} \frac{\pi \hbar^{2}}{m} \simeq \frac{3}{5} \Lambda_{c} \hbar c$, for the non-relativistic limit (where $\Lambda_{c}=\pi \hbar / m c=1.2 \times$ $10^{-10} \mathrm{~cm}$, and $\hbar$ is the Planck constant divided by $2 \pi$ ). While for the electron fluid,

$$
P_{e}=K_{e} n_{e}^{\gamma}
$$

where

$$
\gamma=\alpha ; K_{e}=K_{i}
$$

for non-relativistic limit, and

$$
\gamma=\frac{4}{3} ; K_{e}=\frac{3}{4}\left(\frac{\pi^{2}}{9}\right)^{\frac{1}{3}} \hbar c \simeq \frac{3}{4} \hbar c
$$

in the ultra-relativistic limit $[1-3,7,8]$.

\section{Derivation of K-dV Equations}

To observe the electrostatic perturbations propagating in the ultra-relativistic degenerate dense plasma due to the effect of dispersion by analyzing the outgoing solutions of (1)-(5), we first introduce the stretched coordinates [39]

$$
\begin{gathered}
\zeta=-\varepsilon^{1 / 2}\left(x+V_{p} t\right), \\
\tau=\varepsilon^{3 / 2} t,
\end{gathered}
$$

where $V_{p}$ is the wave phase speed ( $\omega / k$ with $\omega$ being angular frequency and $k$ being the wave number of the perturbation mode), and $\varepsilon$ is a smallness parameter measuring the weakness of the dispersion $(0<\varepsilon<1)$. We then expand $n_{s}, n_{e}, u_{s}$, and $\phi$, in power series of $\varepsilon$ :

$$
\begin{aligned}
& n_{s}=1+\varepsilon n_{s}^{(1)}+\varepsilon^{2} n_{s}^{(2)}+\cdots \\
& n_{e}=1+\varepsilon n_{e}^{(1)}+\varepsilon^{2} n_{e}^{(2)}+\cdots \\
& u_{s}=\varepsilon u_{s}^{(1)}+\varepsilon^{2} u_{s}^{(2)}+\cdots \\
& \phi=\varepsilon \phi^{(1)}+\varepsilon^{2} \phi^{(2)}+\cdots
\end{aligned}
$$

and develop equations in various powers of $\varepsilon$. To the lowest order in $\varepsilon,(1)-(16)$ give $u_{s}^{(1)}=-V_{p} n_{s}^{(1)}$,

$$
\begin{gathered}
n_{p}^{(1)}=\phi^{(1)} /\left(V_{p}^{2}-K_{1}^{\prime}\right), \quad n_{n}^{(1)}=-\beta \phi^{(1)} /\left(V_{p}^{2}-K_{1}^{\prime}\right), \\
n_{e}^{(1)}=\phi^{(1)} / K_{2}^{\prime} \text {, and } V_{p}=\sqrt{K_{2}^{\prime} \frac{(1+\beta)-p \mu-\alpha_{e}}{1-p \mu-\alpha_{n}}+K_{1}^{\prime}}
\end{gathered}
$$

where $K_{1}^{\prime}=\alpha K_{1} /(\alpha-1)$ and $K_{2}^{\prime}=\gamma K_{2} /(\gamma-1)$. The relation $V_{p}=\sqrt{K_{2}^{\prime} \frac{(1+\beta)-p \mu-\alpha_{e}}{1-p \mu-\alpha_{n}}+K_{1}^{\prime}} \quad$ represents the dispersion relation for the dust ion-acoustic type electrostatic waves in the degenerate plasma under consideration.

We are interested in studying the nonlinear propagation of these dispersive dust ion-acoustic type electrostatic waves in a degenerate plasma. To the next higher 
order in $\varepsilon$, we obtain a set of equations

$$
\begin{gathered}
\frac{\partial n_{s}^{(1)}}{\partial \tau}-V_{p} \frac{\partial n_{s}^{(2)}}{\partial \zeta}-\frac{\partial}{\partial \zeta}\left[u_{s}^{(2)}+n_{s}^{(1)} u_{s}^{(1)}\right]=0, \\
\frac{\partial u_{p}^{(1)}}{\partial \tau}-V_{p} \frac{\partial u_{p}^{(2)}}{\partial \zeta}-u_{p}^{(1)} \frac{\partial u_{p}^{(1)}}{\partial \zeta}-\frac{\partial \phi^{(2)}}{\partial \zeta} \\
-K_{1}^{\prime} \frac{\partial}{\partial \zeta}\left[n_{p}^{(2)}+\frac{(\alpha-2)}{2}\left(n_{p}^{(1)}\right)^{2}\right]=0, \\
\frac{\partial u_{n}^{(1)}}{\partial \tau}-V_{p} \frac{\partial u_{n}^{(2)}}{\partial \zeta}-u_{n}^{(1)} \frac{\partial u_{n}^{(1)}}{\partial \zeta}+\beta \frac{\partial \phi^{(2)}}{\partial \zeta} \\
-K_{1}^{\prime} \frac{\partial}{\partial \zeta}\left[n_{n}^{(2)}+\frac{(\alpha-2)}{2}\left(n_{n}^{(1)}\right)^{2}\right]=0, \\
\frac{\partial \phi^{(2)}}{\partial \zeta}-K_{2}^{\prime} \frac{\partial}{\partial \zeta}\left[n_{e}^{(2)}+\frac{(\gamma-2)}{2}\left(n_{e}^{(1)}\right)^{2}\right]=0, \\
\frac{\partial^{2} \phi^{(1)}}{\partial \zeta^{2}}=\left(1-p \mu-\alpha_{n}\right) n_{e}^{(2)}-n_{p}^{(2)}+\left(1-p \mu-\alpha_{e}\right) n_{n}^{(2)} .
\end{gathered}
$$

Now, combining (17)-(21) we deduce a Korteweg-de Vries equation

$$
\frac{\partial \phi^{(1)}}{\partial \tau}+A \phi^{(1)} \frac{\partial \phi^{(1)}}{\partial \zeta}+B \frac{\partial^{3} \phi^{(1)}}{\partial \zeta^{3}}=0
$$

where

$$
\begin{aligned}
A= & \frac{\left(V_{p}^{2}-K_{1}^{\prime}\right)^{2}}{2 V_{p}\left[1+\left(1-p \mu-\alpha_{e}\right)\right] \beta} \\
& \times\left[\frac{\left[3 V_{p}^{2}+K_{1}^{\prime}(\alpha-2)\right]\left[1+\left(1-p \mu-\alpha_{e}\right)\right] \beta^{2}}{\left(V_{p}^{2}-K_{1}^{\prime}\right)^{3}}\right. \\
& \left.+\frac{(\gamma-2)\left(1-p \mu-\alpha_{n}\right) \beta^{2}}{K_{2}^{\prime 2}}\right] \\
B= & \frac{\left(V_{p}^{2}-K_{1}^{\prime}\right)^{2}}{2 V_{p}\left[1+\left(1-p \mu-\alpha_{e}\right)\right] \beta} .
\end{aligned}
$$

The stationary solitary wave solution of (22) is

$$
\phi^{(1)}=\phi_{m} \operatorname{sech}^{2}\left(\frac{\xi}{\Delta}\right)
$$

where $\xi=\zeta-u_{0} \tau, \phi_{m}=3 u_{0} / A$, and $\Delta=\left(4 B / u_{0}\right)^{1 / 2}$. It is obvious from (23) and (35) that the degenerate plasma under consideration supports compressive electrostatic solitary waves which are associated with a positive potential. It is obvious from (23)-(35) that the amplitude $\left[\phi_{m}\right]$ of these solitary structures depends on the density parameter $\mu$, i.e., the ratio of electron to positive ion number density. The electrostatic solitary profiles are shown in Figures 1-4. This is obvious that the profiles are quite different from those obtained from the previous investigation [7-9]. And the potential for non-relativistic degenerate ion fluid and ultra-relativistic degenerate electron fluid is different from that when both the particles follow the same limit.

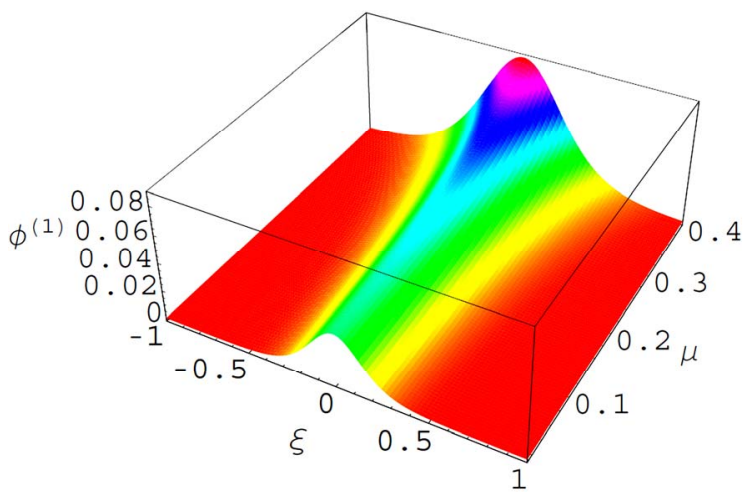

Figure 1. The effect of $\boldsymbol{\mu}$ the potential of solitary wave for both electron and ions (both positive and negative) being non-relativistic degenerate with $p=+1$.

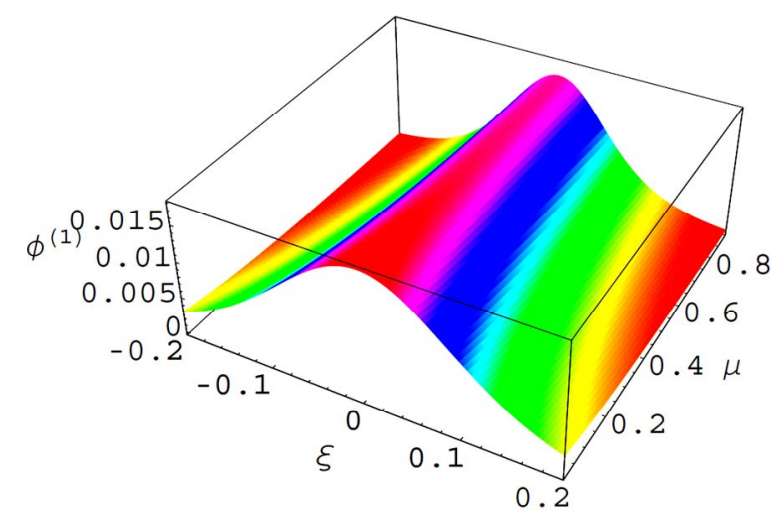

Figure 2. The effect of $\boldsymbol{\mu}$ the potential of solitary wave for both electron and ions (both positive and negative) being non-relativistic degenerate with $p=-1$.

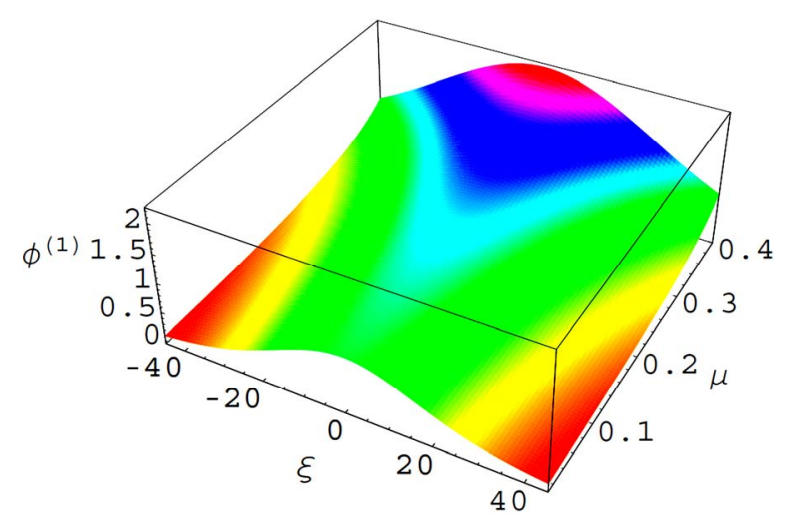

Figure 3. The effect of $\boldsymbol{\mu}$ the potential of solitary wave for electron being ultra-relativistic and ions (both positive and negative) being non-relativistic degenerate with $p=+1$. 


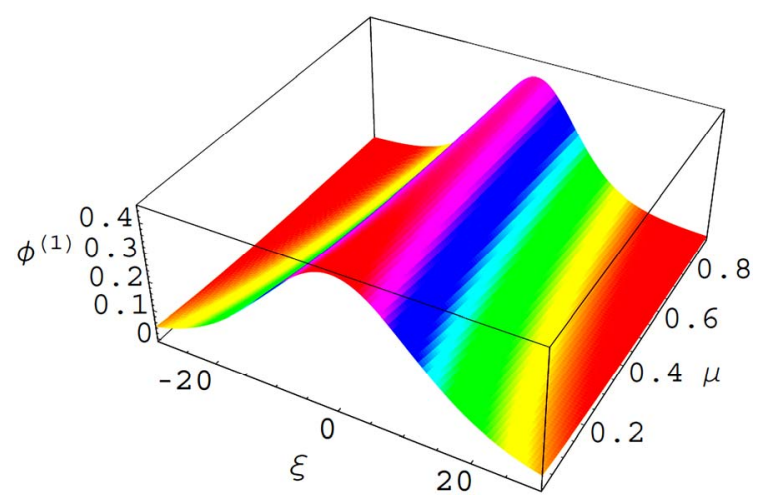

Figure 4. The effect of $\boldsymbol{\mu}$ the potential of solitary wave for electron being ultra-relativistic and ions (both positive and negative) being non-relativistic degenerate with $p=-1$.

Now we are returning to (22) with the term $\phi^{(1)}$ which changes proportionally with the parameter $\mu$ and the polarity, as well as with the sign of $p$. We already have numerically solved (22), and have observed the effects of $\mu$ on electrostatic solitary structures in case of both non-relativistic and ultra-relativistic degenerate electrons (ion always being non-relativistic degenerate). When the number density of the charged dust increases, as well as the parameter $\mu$ is increased, the system potential, which results from the balance between the nonlinearity and dispersion, decreases (increases) when $p$ is positive (negative) which are depicted in Figures 1 and 2. This holds good for the width of solitary profile (shown in Figures 1 and 2). The values of other parameters have been chosen from a standard theoretical analysis [7-9]. For example, when we were investigating the effect of dust number density (i.e., $\mu$ ), other related parameters were kept constant (such as $\alpha_{e}=0.4, \alpha_{n}=0.3$, etc.). For the case of ultra-relativistic degenerate electrons and non relativistic degenerate multi ions, the same effects are observed (shown in Figures 3 and 4).

\section{Derivation of Burger's Equations}

To examine electrostatic perturbations propagating in the relativistic degenerate dense plasma due to the effect of dissipation by analyzing the outgoing solutions of (1)(5), we now introduce the new set of stretched coordinates [40]

$$
\begin{gathered}
\zeta=-\varepsilon\left(x+V_{p} t\right), \\
\tau=\varepsilon^{2} t,
\end{gathered}
$$

To the lowest order in $\varepsilon$, (1)-(10), (13)-(16), (26), and (27) give the same results as we have had for the solitary waves.

To the next higher order in $\varepsilon$, we obtain a set of equations

$$
\begin{aligned}
& \frac{\partial n_{s}^{(1)}}{\partial \tau}-V_{p} \frac{\partial n_{s}^{(2)}}{\partial \zeta}-\frac{\partial}{\partial \zeta}\left[u_{s}^{(2)}+n_{s}^{(1)} u_{s}^{(1)}\right]=0, \\
& \frac{\partial u_{p}^{(1)}}{\partial \tau}-V_{p} \frac{\partial u_{p}^{(2)}}{\partial \zeta}-u_{p}^{(1)} \frac{\partial u_{p}^{(1)}}{\partial \zeta}-\frac{\partial \phi^{(2)}}{\partial \zeta}-\eta \frac{\partial^{2}}{\partial \zeta^{2}} u_{p}^{(1)} \\
& -K_{1}^{\prime} \frac{\partial}{\partial \zeta}\left[n_{p}^{(2)}+\frac{(\alpha-2)}{2}\left(n_{p}^{(1)}\right)^{2}\right]=0, \\
& \frac{\partial u_{n}^{(1)}}{\partial \tau}-V_{p} \frac{\partial u_{n}^{(2)}}{\partial \zeta}-u_{n}^{(1)} \frac{\partial u_{n}^{(1)}}{\partial \zeta}+\beta \frac{\partial \phi^{(2)}}{\partial \zeta}-\eta \frac{\partial^{2}}{\partial \zeta^{2}} u_{n}^{(1)} \\
& -K_{1}^{\prime} \frac{\partial}{\partial \zeta}\left[n_{n}^{(2)}+\frac{(\alpha-2)}{2}\left(n_{n}^{(1)}\right)^{2}\right]=0, \\
& \frac{\partial \phi^{(2)}}{\partial \zeta}-K_{2}^{\prime} \frac{\partial}{\partial \zeta}\left[n_{e}^{(2)}+\frac{(\gamma-2)}{2}\left(n_{e}^{(1)}\right)^{2}\right]=0, \\
& 0=\left(1-p \mu-\alpha_{n}\right) n_{e}^{(2)}-n_{p}^{(2)}+\left(1-p \mu-\alpha_{e}\right) n_{p}^{(2)} .
\end{aligned}
$$

Now, combining (28)-(32) we deduce a Burger's equation

$$
\frac{\partial \phi^{(1)}}{\partial \tau}+A \phi^{(1)} \frac{\partial \phi^{(1)}}{\partial \zeta}=C \frac{\partial^{2} \phi^{(1)}}{\partial \zeta^{2}},
$$

where the value of $A$ is the same as before and $C$ is given by

$$
C=\frac{\eta}{2} .
$$

The shock wave solution of (33) is

$$
\phi^{(1)}=\phi_{m}\left[1-\tanh \left(\frac{\xi}{\delta}\right)\right],
$$

where $\phi_{m}=u_{0} / A$ and $\delta=2 C / u_{0}$.

The electrostatic shock profiles, caused by the balance between nonlinearity and dissipation, are shown in Figures 5-12. The effect of $\xi$ and $\mu$ on potential of the shock wave observed from Figures 5-8 for both of nonrelativistic and ultra-relativistic degenerate electron and non-relativistic degenerate multi-ion dusty plasma in case of both positive and negative charged dust grains. We have also numerically solved Equation (33), and have observed the effects of $\mu$ on electrostatic shock structures in case of both non-relativistic and ultra-relativistic degenerate electrons (ion always being non-relativistic degenerate). When the number density of the charged dust grains increases, as well as the parameter $\mu$ is increased, the system potential, which results from the balance between the nonlinearity and dissipation, decreases (increases) when $p$ is positive (negative) which are depicted in Figures $\mathbf{9}$ and $\mathbf{1 0}$.

The number density of dust grains, as well as $\mu$ has significant effect on the potential of the electrostatic shock waves depending on its charged polarity, as well 


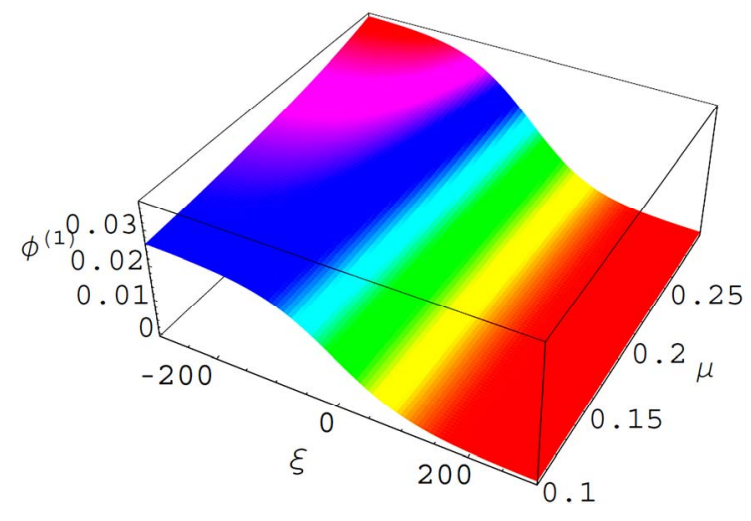

Figure 5. The effect of $\boldsymbol{\mu}$ the potential of solitary wave for both electron and ions (both positive and negative) being non-relativistic degenerate with $p=+1$.

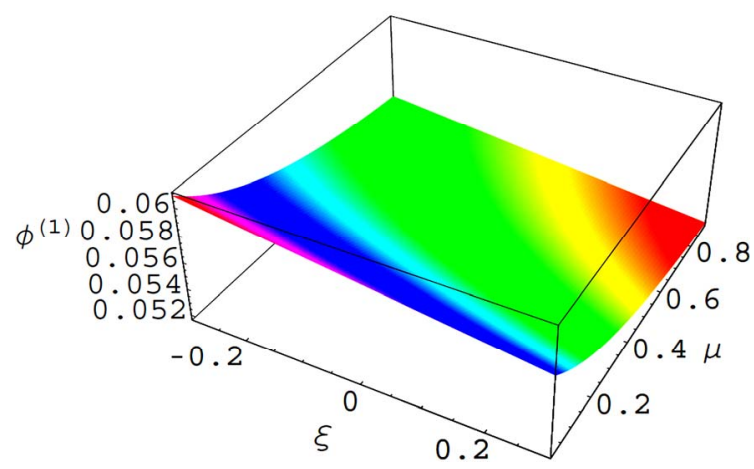

Figure 6. The effect of $\boldsymbol{\mu}$ the potential of solitary wave for both electron and ions (both positive and negative) being non-relativistic degenerate with $p=-1$.

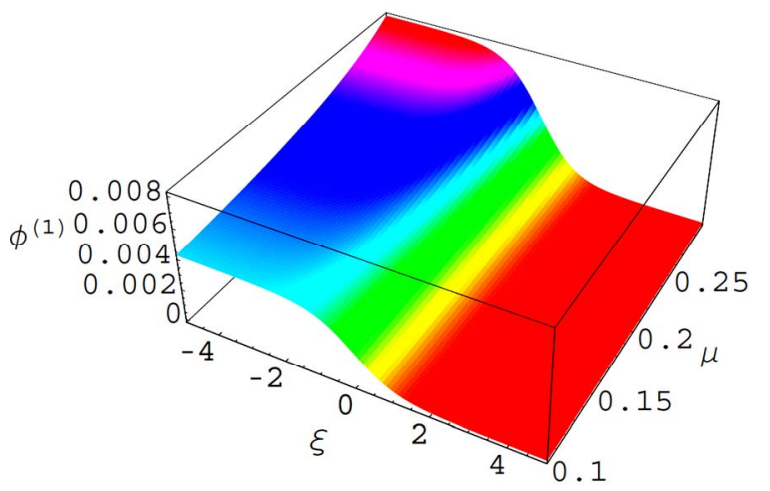

Figure 7. The effect of $\boldsymbol{\mu}$ the potential of solitary wave for electron being ultra-relativistic and ions (both positive and negative) being non-relativistic degenerate with $p=+1$.

as $p$ for ultra-relativistic degenerate electrons and nonrelativistic degenerate multi-ion, the same manner as the previous just discussed which is shown in (11) and (12). So it is clear that the number density of charged dust (as well as $\mu$ ) has a significant effect on the potential, as well as other parameters (like $\eta$ ) of wave profile for non-relativistic degenerate ion fluid and both non-rela- tivistic and ultra-relativistic degenerate electron fluid depending on the polarity of the charged dust grains. All other parameters were kept constant (such as $\alpha_{e}=0.4$, $\alpha_{n}=0.3, u_{0}=0.1$ etc.).

The parameter $\eta$ was chosen from standard value [40] for the system under consideration.

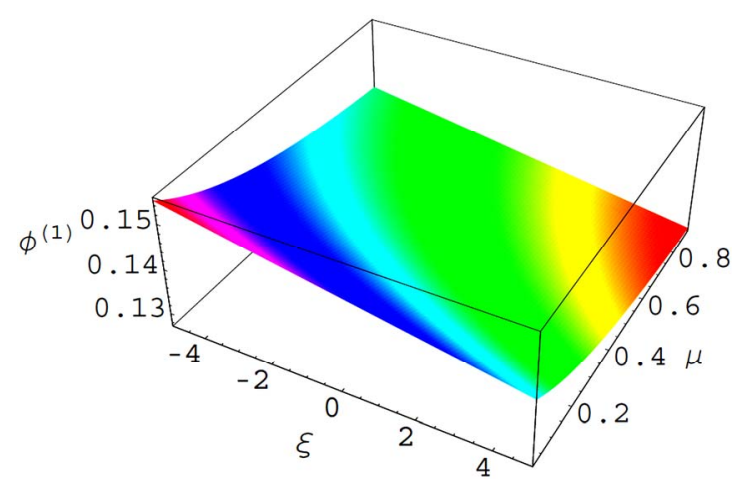

Figure 8. The effect of $\boldsymbol{\mu}$ the potential of solitary wave for electron being ultra-relativistic and ions (both positive and negative) being non-relativistic degenerate with $p=-1$.

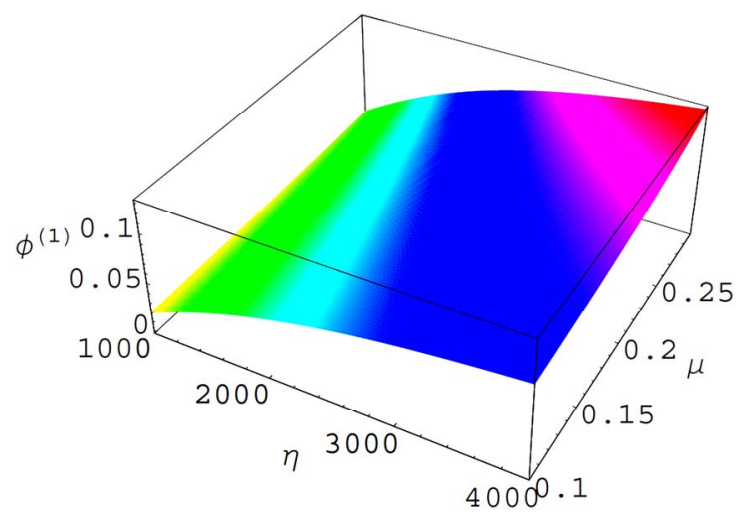

Figure 9. The effect of the variation of $\mu$ and $\eta$ on the potential of solitary wave for both electron and ions (both positive and negative) being non-relativistic degenerate with $p=+1$.

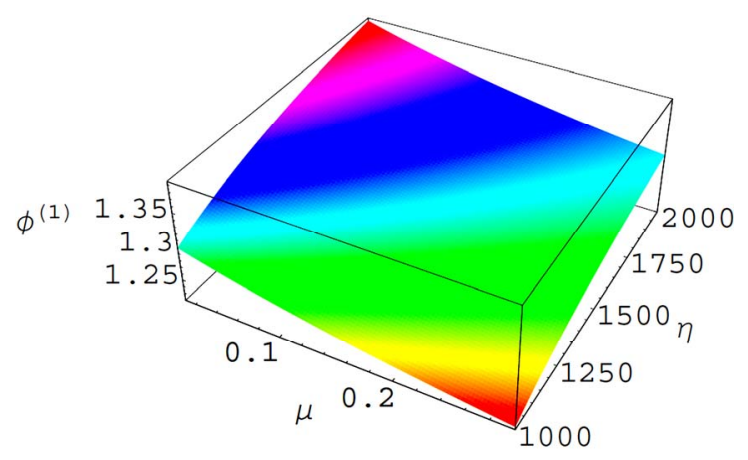

Figure 10. The effect of the variation of $\mu$ and $\eta$ on the potential of solitary wave for both electron and ions (both positive and negative) being non-relativistic degenerate with $p=-1$. 


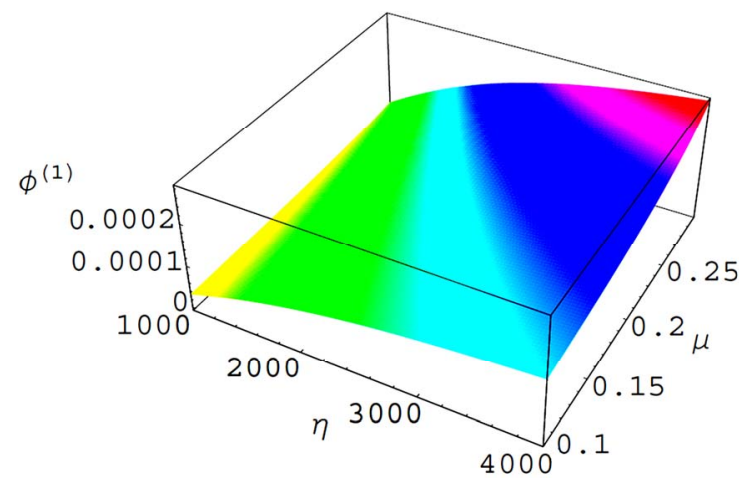

Figure 11. The effect of the variation of $\mu$ and $\eta$ on the potential of solitary wave for electron being ultra-relativistic and ions (both positive and negative) being non-relativistic degenerate with $p=+1$.

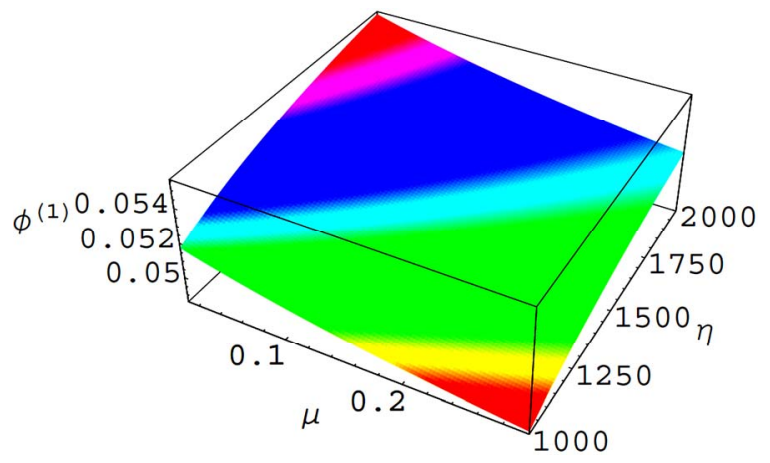

Figure 12. The effect of the variation of $\mu$ and $\eta$ on the potential of solitary wave for electron being ultra-relativistic and ions (both positive and negative) being nonrelativistic degenerate with $p=-1$.

\section{Discussion}

The nonlinear electrostatic perturbation for degenerate dense relativistic multi-ion plasma with additional particles, positive and negative charged dust grains, has been analyzed. The nonlinear electrostatic propagation modes, particularly the solitary and shock profiles has been theoretically studied. It has been observed that the degenerate electron and multi-ion pressures and the number density of the charged dust grains, as well as the charged polarity have significant effects on the potential of the electrostatic profiles (shown in (1)-(12)).

Our recent observation is different from the related investigations [7-9] where we have considered the pressure of all the constituent particles (electrons and multiion), as the plasma system under consideration is degenerate and all the particles should follow the equation of state (6)-(10) whatever the limit is (either non-relativistic or ultra-relativistic), again the effect of arbitrary charged static dust grains. Hence our present investigation is more acceptable and the plasma system constituents have made the validity of our investigation totally different and also greater than the previous works [7-9].
In our numerical analysis we have tried to use a wide range of the degenerate plasma parameters, which are relevant for many cosmic environments and compact astrophysical objects. The results of the present investigation is, therefore, expected to be useful in understanding the dispersion properties of the electrostatic waves in such cosmic environments and compact astrophysical objects [10-17].

\section{Acknowledgements}

The Third World Academy of Science (TWAS) Research Grant for the research equipment is gratefully acknowledged. The constructive suggestions of the anonymous reviewer are also gratefully acknowledged.

\section{REFERENCES}

[1] S. Chandrasekhar, "The Density of White Dwarf Stars," Philosophical Magazine, Vol. 11, No. 7, 1931, pp. 592596.

[2] S. Chandrasekhar, "The Maximum Mass of Ideal White Dwarfs?” Astrophysical Journal, Vol. 74, No. 1, 1931, pp. 81-82. doi:10.1086/143324

[3] S. Chandrasekhar, "The Highly Collapsed Configurations of a Stellar Mass (Second Paper)," Monthly Notices of the Royal Astronomical Society, Vol. 95, 1935, pp. 226-260.

[4] D. Koester and G. Chanmugam, "Physics of White Dwarf Stars," Reports on Progress in Physics, Vol. 53, No. 7, 1990, p. 837. doi:10.1088/0034-4885/53/7/001

[5] S. L. Shapiro and S. A. Teukolsky, "Black Holes, White Dwarfs, and Neutron Stars: The Physics of Compact Objects," Wiley, New York, 1983.

[6] E. Garcia-Berro, S. Torres, L. G. Althaus, I. Renedo, P. Lorén-Aguiltar, A. H. Córsico R. D. Rohrmann, M. Salaris and J. Isern, "A White Dwarf Cooling Age of 8 Gyr for NGC 6791 from Physical Separation Processes," Nature, Vol. 465, 2010, pp. 194-196.

[7] A. A. Mamun and P. K. Shukla, "Nonplanar Dust Ion-Acoustic Solitary and Shock Waves in a Dusty Plasma with Electrons Following a Vortex-Like Distribution," Physics Letter A, Vol. 374, No. 3, 2010, pp. 472457. doi:10.1016/i.physleta.2009.08.071

[8] A. A. Mamun and P. K. Shukla, "Solitary Waves in an Ultrarelativistic Degenerate Dense Plasma," Physics of Plasmas, Vol. 17, No. 10, 2010, Article ID: 104504.

[9] N. Roy, S. Tasnim and A. A. Mamun, "Solitary Waves and Double Layers in Degenerate Dusty Electron-Positron-Ion Plasma," Physics of Plasmas, Vol. 19, No. 6, 2012, Article ID: 064704. doi:10.1063/1.4725497

[10] F. C. Michel, "Theory of Pulsar Magnetospheres," Reviews of Modern Physics, Vol. 54, No. 1, 1982, pp. 1-66. doi:10.1103/RevModPhys.54.1

[11] S. L. Shapiro and S. A. Teukolsky, "Black Holes, White Dwarfs and Neutron Stars: The Physics of Compact Objects," John Wiley and Sons, New York, 1983.

[12] M. Y. Yu, P. K. Shukla and L. Stenflo, “Alfven Vortices 
in a Strongly Magnetized Electron-Position Plasma", Astrophysical Journal, Vol. 309, 1986, pp. L63-L65. doi:10.1086/184761

[13] P. K. Shukla, N. N. Rao, M. Y. Yu and N. L. Tsintsadze, "Relativistic Nonlinear Effects in Plasmas Review Articl," Physics Reports, Vol. 138, No. 1-2, 1986, pp. 1-149. doi:10.1016/0370-1573(86)90157-2

[14] H. R. Miller and P. J. Witta, "Active Galactic Nuclei," Springer, New York, 1987.

[15] E. Tandberg-Hansen and A. G. Emslie, "The Physics of Solar Flares," Cambridge University Press, Cambridge, 1988.

[16] L. O. Silva, R. Bingham, J. M. Dawson, J. T. Mendona and P. K. Shukla, "Neutrino Kinetics in Dense Astrophysical Plasmas," Physics Review Letter, Vol. 83, No. 14, 1999, pp. 2703-2706. doi:10.1103/PhysRevLett.83.2703

[17] J. Hoyos, A. Reisenegger and J. A. Valdivia, "Magnetic Field Evolution in Neutron Stars: One-Dimensional MultiFluid Model," Astronomy \& Astrophysics, Vol. 487, No. 3, 2008, pp. 789-803. doi:10.1051/0004-6361:200809466

[18] V. P. Bliokh and V. V. Yaroshenko, Soviet Astronomy, Vol. 29, 1985, p. 330.

[19] U. de Angelisa, V. Formisanoa and M. Giordanoa, "Ion Plasma Waves in Dusty Plasmas: Halley's Comet," Journal of Plasma Physics, Vol. 40, 1988, p. 399.

[20] P. K. Shukla and V. P. Silin, "Low-Frequency Modes in Dusty Plasmas," Physica Scripta, Vol. 45, No. 5, 1992, p. 504. doi:10.1088/0031-8949/45/5/014

[21] M. J. Rees, "The Very Early Universe," Cambridge University Press, Cambridge, 1983.

[22] H. R. Miller and P. J. Witta, "Active Galactic Nuclei," Springer, New York, 1987.

[23] F. C. Michel, "Theory of Pulsar Magnetospheres," Review of Modern Physics, Vol. 54, No. 1, 1982, pp. 1-66. doi:10.1103/RevModPhys.54.1

[24] E. Tandberg-Hansen and A. G. Emslie, "The Physics of Solar Flares," Cambridge University Press, Cambridge, 1988.

[25] T. Piran, "Gamma-Ray Bursts and the Fireball Model," Physics Report, Vol. 314, No. 6, 1999, pp. 575-667. doi:10.1016/S0370-1573(98)00127-6

[26] G. Manfredi, "How to Model Quantum Plasmas," The Fields Institute Communications Series, Vol. 46, 2005, p. 263.

[27] L. Stenfo, P. K. Shukla and M. Marklund, "New LowFrequency Oscillations in Quantum Dusty Plasmas," Eu- rophysics Letter, Vol. 74, No. 5, 2006, p. 844. doi:10.1209/epl/i2006-10032-x

[28] P. K. Shukla, "New Dust in Quantum Plasma," Physics Letter A, Vol. 352, No. 3, 2006, pp. 242-243. doi:10.1016/j.physleta.2005.11.065

[29] P. K. Shukla and L. Stenfo, "Jeans Instabilities in Quantum Dusty Plasmas," Physics Letter A, Vol. 355, No. 4-5, 2006, pp. 378-380. doi:10.1016/j.physleta.2006.02.054

[30] G. Brodin and M. Marklund, "Spin Magnetohydrodynamics," New Journal of Physics, Vol. 9, No. 8, 2007, p. 227. doi:10.1088/1367-2630/9/8/277

[31] G. Brodin and M. Marklund, "Spin Solitons in Magnetized Pair Plasmas," Physics of Plasmas, Vol. 14, No. 11, 2007, Article ID: 112107. doi:10.1063/1.2793744

[32] M. Marklund and G. Brodin, "Dynamics of Spin-1/2 Quantum Plasmas," Physics Review Letter, Vol. 98, No. 2, 2007, Article ID: 025001. doi:10.1103/PhysRevLett.98.025001

[33] M. Marklund, B. Eiasson and P. K. Shukla, "Magnetosonic Solitons in a Fermionic Quantum Plasma," Physics Review E, Vol. 76, No. 6, 2007, Article ID: 067401. doi:10.1103/PhysRevE.76.067401

[34] P. K. Shukla and B. Eliasson, "Nonlinear Aspects of Quantum Plasma Physics" Physics-Uspekhi, Vol. 53, No. 1, 2010, p. 51. doi:10.3367/UFNe.0180.201001b.0055

[35] W. Masood, B. Eiasson and P. K. Shukla, "Electromagnetic Wave Equations for Relativistically Degenerate Quantum Magnetoplasmas," Physics Review E, Vol. 81, No. 6, 2010, Article ID: 066401. doi:10.1103/PhysRevE.81.066401

[36] F. Hass, "Variational Approach for the Quantum Zakharov System," Physics of Plasmas, Vol. 14, No. 4, 2007, Article ID: 042309.

[37] A. Misra and S. Samanta, "Quantum Electron-Acoustic Double Layers in a Magnetoplasma," Physics of Plasmas, Vol. 15, No. 12, 2008, Article ID: 122307. doi:10.1063/1.3040014

[38] A. P. Misra, S. Banerjee, F. Haas, P. K. Shukla, and L. P. G. Assis, "Temporal Dynamics in the One-Dimensional Quantum Zakharov Equations for Plasmas," Physics of Plasmas, Vol. 17, No. 3, 2010, Article ID: 032307. doi:10.1063/1.3356059

[39] S. Maxon and J. Viecelli, "Spherical Solitons," Physics Review Letter, Vol. 32, No. 1, 1974, pp. 4-6. doi:10.1103/PhysRevLett.32.4

[40] A. Gavrikov, et al., "Heat Transfer in Dusty Plasma," 32nd EPS Conference on Plasma Physics, Tarragona, 7 June-1 July 2005. 